\title{
ESTROGEN RECEPTOR BINDING AFFINITY OF FOOD CONTACT MATERIAL COMPONENTS ESTIMATED BY QSAR
}

\author{
Jitka Sosnovcová, Marián Rucki, Hana Bendová \\ National Institute of Public Health, Prague, Czech Republic
}

\begin{abstract}
SUMMARY
Aim: The presented work characterized components of food contact materials (FCM) with potential to bind to estrogen receptor (ER) and cause adverse effects in the human organism.

Methods: The QSAR Toolbox, software application designed to identify and fill toxicological data gaps for chemical hazard assessment, was used. Estrogen receptors are much less of a lock-and-key interaction than highly specific ones. The ER is nonspecific enough to permit binding with a diverse array of chemical structures. There are three primary ER binding subpockets, each with different requirements for hydrogen bonding.

Results: More than 900 compounds approved as of FCM components were evaluated for their potential to bind on ER. All evaluated chemicals were subcategorized to five groups with respect to the binding potential to ER: very strong, strong, moderate, weak binder, and no binder to ER. In total 46 compounds were characterized as potential disturbers of estrogen receptor.

Conclusion: Among the group of selected chemicals, compounds with high and even very high affinity to the ER binding subpockets were found. These compounds may act as gene activators and cause adverse effects in the organism, particularly during pregnancy and breast-feeding. It should be considered to carry out further in vitro or in vivo tests to confirm their potential to disturb the regulation of physiological processes in humans by abnormal ER signaling and subsequently remove these chemicals from the list of approved food contact materials.
\end{abstract}

Key words: QSAR, estrogen receptor, chemicals, food contact materials

Address for correspondence: M. Rucki, National Institute of Public Health, Šrobárova 48, 10042 Prague 10, Czech Republic. E-mail: marian. rucki@szu.cz

http://dx.doi.org/10.21101/cejph.a4813

\section{INTRODUCTION}

In 1958 Elwood Jensen demonstrated the existence of an estrogen receptor (ER) (1), the corresponding gene was cloned in 1985. In 1996 estrogen receptor beta $(\mathrm{ER} \beta)$ was cloned from the rat prostate and ovary (2). Reproductive tissues (uterus, ovary), breast, kidney, bone, white adipose tissue, and liver are the places where estrogen receptor alfa $(\mathrm{ER} \alpha)$ is mainly expressed, while expression of ER $\beta$ is found in the ovary, central nervous system (CNS), cardiovascular system, lung, male reproductive organs, prostate, colon, kidney, and the immune system. ERs are found mainly in the nucleus, but also in the cytoplasm and mitochondria.

The ER $\alpha$ and ER $\beta$ genes are located on different chromosomes, $6 q 25.1$ and 14q23.2, respectively. ERs are composed of three functional domains: the NH2-terminal domain (NTD), the DNAbinding domain (DBD), and the $\mathrm{COOH}$-terminal ligand-binding domain (LBD). The NTD encompasses a ligand independent activation function (AF1) domain involved in transcriptional activation of target genes, and with only $16 \%$ similarity between ER $\alpha$ and ER $\beta$. The DBD is highly conserved between ER $\alpha$ and ER $\beta$ with $97 \%$ amino acid identity and mediates sequence-specific binding of ERs to DNA sequences in target genes denoted estrogen-responsive elements (EREs). In contrast, the LBDs of
ER $\alpha$ and ER $\beta$ show a $59 \%$ overall amino acid sequence identity yet the ligand-binding pockets of the two subtypes show only minor differences in structure (3).

Estrogens have been distinctly shown to regulate glucose and lipid metabolism using either models of estrogen-/ER-depletion or estrogen application/replacement. Estrogen deficiency promotes metabolic dysfunction predisposing to type 2 diabetes (T2D), obesity, and the metabolic syndrome. In rodents it has been demonstrated that aromatase is the key enzyme of estrogen production. Knockout (ArKO) mice display insulin resistance (IR), impaired glucose tolerance (IGT), and increased abdominal fat, which are reversible by $17 \beta$-estradiol (E2) treatment (4).

The ER is nonspecific enough to permit binding with a diverse array of chemical structures. There are four primary ER binding subpockets $(5,6)$, each with different requirements for hydrogen bonding (Fig. 1-3). Steroidal compounds usually interact at two points within the ER using two hydrogen-bonding groups. However, there are also chemicals with one hydrogen-bonding group that bind to the ER and cause subsequent gene activation.

The aim of the work was to characterize chemicals with potential to bind to ER and cause adverse effects in the organism. Over nine hundred compounds used in food contact materials (EU Regulation No 10/2011 on plastic materials and articles) (7) were analyzed using QSAR and computational chemistry. 




Fig. 1. A-B interaction of compound with $E R$ binding site (17 $\beta$-Estradiol).

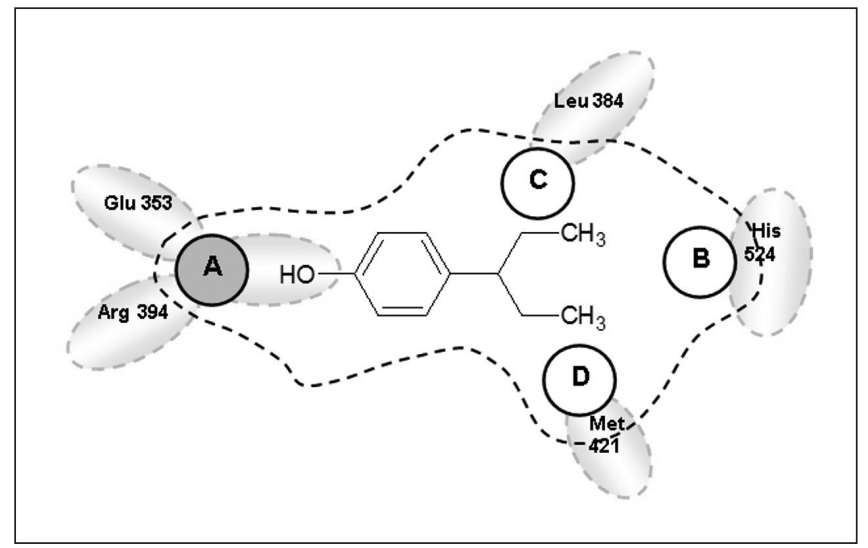

Fig. 2. A-site only interaction of compound with ER binding site.

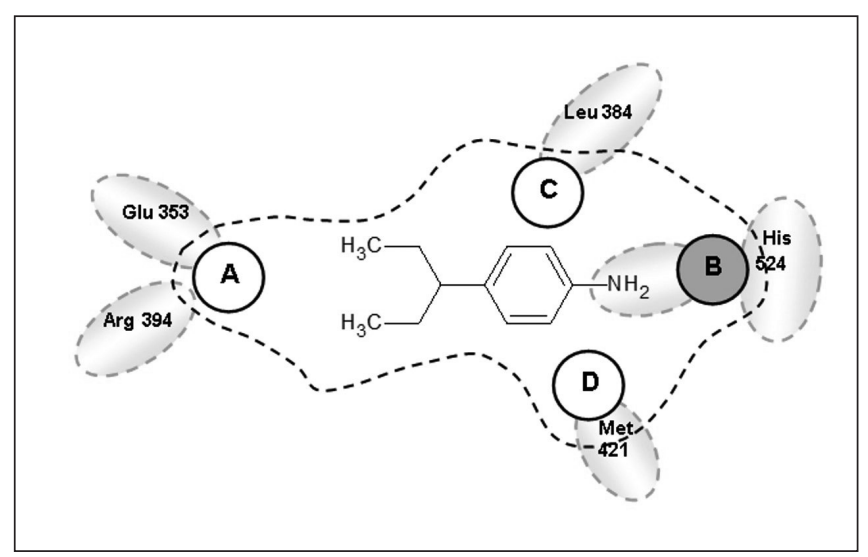

Fig. 3. B-site only interaction of compound with ER binding site.

\section{MATERIALS AND METHODS}

The QSAR Toolbox software application designed to identify and fill toxicological data gaps for chemical hazard assessment, was used *

Compounds were subcategorized to groups using cascade of queries. The first query in the tree is whether the chemical contains a cycle. If not, the chemical is considered within the domain of the ER binding model, but at the same time the chemical is predicted to be inactive in the ER-mediated pathway. The second query is aimed to determine if the chemical belongs to one of the subgroups in which all tested members so far did not competitively bind to ER. For the majority of chemicals, the presence of a charge in the molecule, absence of hydrogen bonding group, or inappropriate geometry explains the failure of these chemicals to bind to ER. Chemicals which meet third query requirements are these with greatest binding affinity to the ER receptors. The primary feature for high ER binding activity is to have two oxygen atoms available for hydrogen bonding at the specific distances dictated by the ER receptor. Molecular weight and partition coefficient of evaluated compounds are also considered.

More than 900 compounds from the list of FCM were evaluated on their potential to bind on ER using QSAR Toolbox.

\section{RESULTS}

All evaluated chemicals were subcategorized to five groups with respect to the binding potential to ER: very strong, strong, moderate, weak binder, and no binder to ER. In total 46 compounds were characterized as potential disturbers of estrogen receptor (Table 1).

The group of very strong binders to ER includes 7 chemicals. These compounds can significantly disturb the ER and can cause number of adverse effects in the organism. Their presence in the list of food contact materials should be questioned, evaluated and proper decision should be adopted. These substances represent a real threat to the health of the population, especially for children and nursing mothers.

The group of strong binders to ER includes 13 chemicals. These compounds can also significantly disturb the ER and may cause a number of adverse effects in the organism. Their presence in the list of food contact materials should be also questioned and re-evaluated as these substances may also represent a potential threat to the health of the sensitive population.

The group of moderate binders to ER includes 7 chemicals. These compounds have a limited potential to disturb the ER and may also cause adverse effects in the organism. Although the total effect is not so strong, continuous disturbing of the ER can lead to chronic problems with human health where the reason is not known.

The group of weak binders to the ER includes 19 chemicals. These compounds have a weak potential to disturb the ER. Their effect on human health is rather questionable, only in case of very high exposition they can significantly disturb the ER.

\section{DISCUSSION}

Estrogen receptors alpha and beta are transcription factors that are involved in the regulation of many complex physiological processes in humans. Abnormal ER signaling leads to development of a variety of diseases, such as cancer, metabolic and cardiovascular disease, neurodegeneration, inflammation (8), and osteoporosis (3). Chemical binding to the ER is one of the significant mechanisms interfering with process of reproduction $(9,10)$.

\footnotetext{
*http://www.qsartoolbox.org/home
} 
Table 1. The list of evaluated chemicals showing affinity to the ER binding site

\begin{tabular}{|c|c|c|c|}
\hline No & CAS No & Substance name & ER binding affinity \\
\hline 1 & $59-02-9$ & a-tocopherol & WA \\
\hline 2 & $69-72-7$ & salicylic acid & WA \\
\hline 3 & $80-05-7$ & 2,2-bis(4-hydroxyphe-nyl)propane (Bisphenol A) & VSA \\
\hline 4 & $80-09-1$ & 4,4'-dihydroxydiphenyl sulphone (Bisfenol S) & MA \\
\hline 4 & $84-74-2$ & phthalic acid, dibutyl ester & MA \\
\hline 6 & $85-68-7$ & phthalic acid, benzyl butyl ester & MA \\
\hline 7 & $87-18-3$ & salicylic acid, 4-tert-butylphenyl ester & SA \\
\hline 8 & $88-68-6$ & 2-aminobenzamide & WA \\
\hline 9 & $92-88-6$ & 4,4'-dihydroxybiphenyl & MA \\
\hline 10 & $94-13-3$ & 4-hydroxybenzoic acid, propyl ester & MA \\
\hline 11 & $95-48-7$ & 0-cresol & WA \\
\hline 12 & $96-69-5$ & 4,4'-thiobis(6-tert-butyl-3-methylphenol) & VSA \\
\hline 13 & $97-23-4$ & 2,2'-dihydroxy--5,5'-dichlorodiphenyl-methane & VSA \\
\hline 14 & $97-53-0$ & eugenol & WA \\
\hline 15 & $98-54-4$ & 4-tert-butylphenol & WA \\
\hline 16 & $99-76-3$ & 4-hydroxybenzoic acid, methyl ester & WA \\
\hline 17 & 99-96-7 & p-hydroxybenzoic acid & WA \\
\hline 18 & $103-90-2$ & $\mathrm{~N}$-(4-hydroxyphenyl) acetamide & WA \\
\hline 19 & $106-44-5$ & p-cresol & WA \\
\hline 20 & $108-39-4$ & m-cresol & WA \\
\hline 21 & $108-45-2$ & 1,3-phenylenediamine & WA \\
\hline 22 & $108-46-3$ & 1,3-dihydroxybenzene & WA \\
\hline 23 & $108-91-8$ & cyclohexylamine & WA \\
\hline 24 & $108-95-2$ & phenol & WA \\
\hline 25 & $119-36-8$ & salicylic acid, methyl ester & WA \\
\hline 26 & $120-47-8$ & 4-hydroxybenzoic acid, ethyl ester & WA \\
\hline 27 & $120-80-9$ & 1,2-dihydroxybenzene & WA \\
\hline 28 & $121-79-9$ & gallic acid, propyl ester & SA \\
\hline 29 & $123-31-9$ & 1,4-dihydroxybenzene & WA \\
\hline 30 & $131-53-3$ & 2,2'-dihydroxy-4-methoxybenzophenone & VSA \\
\hline 31 & $131-56-6$ & 2,4-dihydroxybenzophenone & SA \\
\hline 32 & $131-57-7$ & 2-hydroxy-4-methoxybenzophenone & SA \\
\hline 33 & $599-64-4$ & 4-cumylphenol & SA \\
\hline 34 & $611-99-4$ & 4,4'-dihydroxybenzophenone & VSA \\
\hline 35 & $1034-01-1$ & gallic acid, octyl ester & SA \\
\hline 36 & $1166-52-5$ & gallic acid, dodecyl ester & SA \\
\hline 37 & $1761-71-3$ & bis(4-aminocyclohexyl)methane & SA \\
\hline 38 & $1843-05-6$ & 2-hydroxy-4-n-octyloxybenzophenone & SA \\
\hline 39 & $2440-22-4$ & 2-(2'-hydroxy-5'-methylphenyl)benzotriazole & SA \\
\hline 40 & $2855-13-2$ & 1-amino-3-aminomethyl-3,5,5-trimethyl-cyclohexane & MA \\
\hline 41 & $3293-97-8$ & 2-hydroxy-4-n-hexyloxybenzophenone & SA \\
\hline 42 & $6864-37-5$ & 3,3'-dimethyl-4,4'-diaminodicyclohexylmethane & SA \\
\hline 43 & 25013-16-5 & tert-butyl-4-hydroxyanisole & MA \\
\hline 44 & $27955-94-8$ & 1,1,1-tris(4-hydroxyphenol)ethane & VSA \\
\hline 45 & $47465-97-4$ & 3,3-bis(3-methyl-4-hydroxyphenyl)2-indolinone & VSA \\
\hline 46 & $147315-50-2$ & 2-(4,6-diphenyl-1,3,5-triazin-2-yl)-5-(hexyloxy)phenol & SA \\
\hline
\end{tabular}

WA - weak affinity, MA - medium affinity, SA - strong affinity, VSA - very strong affinity 
Because of the complexity of the effect of potential ER disturbers, the corresponding in vivo method are time consuming and very expensive, especially in the case of reproductive toxicity studies (Extended One-Generation Reproductive Toxicity Study OECD 443) (11). Fast screening methods or in silico methods are the proper way for testing of hundreds of chemicals to find the group of compounds with the highest potential to cause adverse effect in humans. The QSAR Toolbox is one of the most promising computational software in the field of fast, precious and transparent assessment of variety outcomes. It employs the databases of experimental results, trend analysis and proper categorization of compound to the corresponding group and creates well defined QSAR models with applicability domain.

Compounds used in food contact materials represent one of the most important sources of potential contaminants of foodstuffs. They can freely migrate from the packing material to the meal and then can expose humans. Especially in the case of ER disruptors, the effective concentration of acting compounds could be very low, making these compounds even more dangerous.

\section{CONCLUSION}

Based on the measured data and proper categorization the compounds can be placed into groups of inactive chemicals, into "drug-like" groups of chemicals which have high potential for significant ER binding affinity, or into groups of chemicals which may have weak to moderate binding affinity depending on specific properties or structural features.

Over nine hundred compounds used in food contact materials were analyzed using QSAR and computational chemistry. Among the group of selected chemicals, compounds with high and even very high affinity to the ER binding subpockets were found. These compounds may act as gene activators and cause adverse effects in the organism, particularly during pregnancy and breast-feeding. It should be considered to carry out further in vitro or in vivo tests to confirm their potential to disturb the regulation of physiological processes in humans by abnormal ER signaling and subsequently remove these chemicals from the list of food contact materials.

\section{Acknowledgement}

The research was financially supported by the Internal Grant Agency of the Ministry of Health of the Czech Republic (project no. NT 133414/2012).

\section{Conflict of Interests}

None declared

\section{REFERENCES}

1. Jensen EV, Jacobson HI, Flesher JW, Saha NN, Gupta GN, Smith S, et al. Estrogen receptors in target tissues. In: Pincus G, Nakao T, Tait JF, editors. Steroid dynamics. New York: Academic Press; 1966. p. 133-56.

2. Kuiper GG, Enmark E, Pelto-Huikko M, Nilsson S, Gustafsson JA. Cloning of a novel receptor expressed in rat prostate and ovary. Proc Natl Acad Sci U S A. 1996 Jun 11;93(12):5925-30.

3. Jia M, Dahlman-Wright K, Gustafsson JÅ. Estrogen receptor alpha and beta in health and disease. Best Pract Res Clin Endocrinol Metab. 2015 Aug;29(4):557-68.

4. Jones ME, Thorburn AW, Britt KL, Hewitt KN, Wreford NG, Proietto J, et al. Aromatase-deficient (ArKO) mice have a phenotype of increased adiposity. Proc Natl Acad Sci U S A. 2000 Nov 7;97(23):12735-40.

5. Katzenellenbogen J, Muthyala R, Katzenellenbogen BS. The nature of the ligand-binding pocket of estrogen receptor alpha and beta: The search for subtype-selective ligands and implications for the prediction of estrogenic activity. Pure Appl Chem. 2003;75(11-12):2397-403.

6. Asim M, El-Salfiti M, Qian Y, Choueiri C, Salari S, Cheng J, et al. Deconstructing estradiol: removal of B-ring generates compounds which are potent and subtype-selective estrogen receptor agonists. Bioorg Med Chem Lett. 2009 Feb 15;19(4):1250-3.

7. EUR-Lex. Commission Regulation (EU) No 10/2011 of 14 January 2011 on plastic materials and articles intended to come into contact with food [Internet]. EUR-Lex; 2011 [cited 2016 May 20]. Available from: http://eur-lex.europa.eu/legal-content/EN/ALL/?uri=CELEX:0201 1R0010-20150226.

8. Kovats S. Estrogen receptors regulate innate immune cells and signaling pathways. Cell Immunol. 2015 Apr;294(2):63-9.

9. Schmieder PK, Tapper MA, Denny JS, Kolanczyk RC, Sheedy BR, Henry $\mathrm{TR}$, et al. Use of trout liver slices to enhance mechanistic interpretation of estrogen receptor binding for cost-effective prioritization of chemicals within large inventories. Environ Sci Technol. 2004 Dec 1;38(23):633342.

10. Bondesson M, Hao R, Lin CY, Williams C, Gustafsson JÅ. Estrogen receptor signaling during vertebrate development. Biochim Biophys Acta. 2015 Feb;1849(2):142-51.

11. Test no. 443: Extended One-Generation Reproductive Toxicity Study. OECD Guidelines for the testing of chemicals, section 4 Health effects [Internet]. Paris: OECD; 2012 [cited 2016 May 20]. Available from: http://www.oecd-ilibrary.org/environment/test-no-443-extended-onegeneration-reproductive-toxicity-study_9789264185371-en.

Received April 27, 2016 Accepted in revised form September 2, 2016 